\title{
High-resolution Calorimetric Study of a Liquid Crystalline Organo-Siloxane Tetrapode with a Biaxial Nematic Phase
}

\author{
George Cordoyiannis, ${ }^{1}$ Daniela Apreutesei, ${ }^{2}$ Georg H. Mehl, ${ }^{2}$ Christ Glorieux, ${ }^{1}$ and Jan Thoen ${ }^{1, *}$ \\ ${ }^{1}$ Laboratorium voor Akoestiek en Thermische Fysica, Departement Natuurkunde en Sterrenkunde, \\ Katholieke Universiteit Leuven, Celestijnenlaan 200D, B-3001 Leuven, Belgium \\ ${ }^{2}$ Department of Chemistry, The University of Hull, \\ Cottigham Road, Hull HU6 7RX, United Kingdom
}

(Dated: July 10, 2008)

\begin{abstract}
High-resolution adiabatic scanning calorimetry and differential scanning calorimetry have been employed to study the thermal behaviour of an organo-siloxane tetrapode reported to exhibit a biaxial nematic phase. No signature of the uniaxial to biaxial nematic phase transition could be retraced in sequential heating and cooling runs under different scanning rates, within the experimental resolution. The results obtained in this work reveal that an extremely small heat should be involved in the uniaxial to biaxial nematic phase transition. The isotropic to uniaxial nematic transition at $318 \pm 0.01 \mathrm{~K}$ is very stable and it is weakly first order with a rather small latent heat of $0.20 \pm 0.02 \mathrm{~J} / \mathrm{g}$.
\end{abstract}

PACS numbers: 07.20.Fw, 64.70.M-

\section{INTRODUCTION}

The existence of the biaxial nematic phase was theoretically predicted in 1970 by Freiser [1], in terms of a generalised Maier-Saupe theory. Theoretical phase diagrammes were proposed right after by Alben [2] and Straley [3] and the first experimental proof appeared a few years later for a lyotropic system [4]. On the contrary, in thermotropic systems the situation remained elusive for many years [5]. In the late $90 \mathrm{~s}$, some reports on biaxiality appeared, but only in samples sandwiched between glass plates or in free standing films $[6,7]$. It was only in the last five years that new reports on thermotropic biaxiality came out, for liquid crystalline polymers $[8,9]$, bow-shaped liquid crystals [10-15] and liquid-crystalline organo-siloxane tetrapodes [16-18].

The first experimental indications of thermotropic biaxiality attracted immediately a significant interest on this field of research [19], also because of the high potential for applications. The orientation of the secondary director exhibits remarkably improved response times to an applied external field, compared to the ones of the primary director [20, 21]. Illatively, the existence of a thermotropic biaxial nematic phase in ambient temperatures is expected to give a new boost in the liquid crystal display technology. Simultaneously with the experimental hunting of thermotropic biaxiality, a great interest raised for theoretical and simulation studies on systems exhibiting Isotropic $(I)$, uniaxial Nematic $\left(N_{U}\right)$ and biaxial Nematic $\left(N_{B}\right)$ phases [22-32].

Any kind of direct information concerning the type of the $N_{U}-N_{B}$ phase transition (eg. first or second order, universality class) is completely absent from the litera-

\footnotetext{
*jan.thoen@fys.kuleuven.be
}

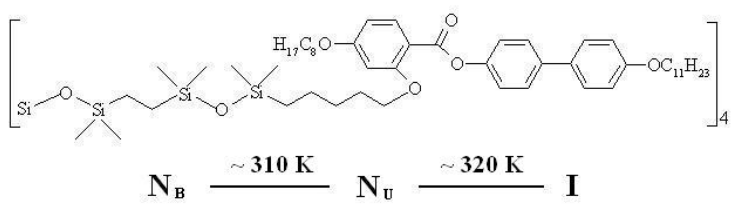

FIG. 1: Molecular structure, phase sequence and transition temperatures of the DW-16 compound.

ture so far. High-resolution calorimetric measurements can enlighten the way to this point and, moreover, they can be employed to explore the fascinating theoreticallyproposed phase diagrammes. In some initial studies these diagrammes were considered to have a special kind of critical point, where two lines of second order transitions merge with a line of first order ones in a sharp cusp $[2,3]$. In recent studies, they are expected to include a tricritical point where a line of second order transitions meets a line of first order ones and a Landau (triple) point where all three phases, $I, N_{U}$ and $N_{B}$ coexist in equilibrium $[22,26,30]$. Consequently, apart from the great interest for applications, the existence of a thermotropic $N_{B}$ phase and the study of the $N_{U}-N_{B}$ transition is also intriguing from the fundamental point of view.

\section{MATERIALS AND METHODS}

The compound under investigation is an organosiloxane tetrapode, namely the compound A in ref. [16] and it was synthesised in the Chemistry Department in the University of Hull. Further down we will refer to it as DW-16. Its chemical structure and phase sequence are shown in Figure 1. Each molecule of DW-16 consists of four (asymmetric) mesogens connected to a siloxane core through spacers. Infrared (IR) absorbance and 
conoscopic measurements have shown for this tetrapode an $I-N_{U}$ transition at $\sim 320 \mathrm{~K}$ and a $N_{U}-N_{B}$ transition at $\sim 310 \mathrm{~K}[16]$.

In this work, we employed high-resolution adiabatic scanning calorimetry (ASC) and differential scanning calorimetry (DSC) to perform a thorough investigation of the thermal behaviour of DW-16. The thermal properties were initially investigated using a Mettler Toledo differential scanning calorimeter (DSC $822^{e}$ ) in nitrogen against an indium standard. Transition temperatures were determined as the onset of the maximum in the endotherm or exotherm. The ASC measurements were performed in a calorimeter consisting of four stages. The inner stage is a cylindrical cell, and it is surrounded by three outer shields. The space between the cell and the shields is vacuum-pumped in order to achieve excellent thermal insulation between them. ASC yields both the heat capacity $\left(C_{p}\right)$ and the enthalpy $(H)$ temperature dependence. It can easily and reliably distinguish between first order and second order phase transitions and reveal subtle features of $C_{p}(T)$. A detailed description of our apparatus can be found elsewhere [33, 34]. For our measurements we used a $10 \mathrm{~g}$ Molybdenum cell, that contained $0.237 \mathrm{~g}$ of DW-16. In order to eliminate any possible temperature gradients in the the sample, a small molybdenum stirring ball was inserted in the cell. Then, by periodically changing the inclination of the whole apparatus (via an automatised mechanism), the stirring ball was moving back and forth inside the cylindrical cell during the experiments. The heat capacity of the empty cell, which was measured in one separate controlexperiment, was subtracted and the result was divided by the sample mass in order to obtain the net specific heat capacity of the sample.

\section{RESULTS AND DISCUSSION}

In this section the results for sequential runs using DSC and ASC are reported. The initial thermal investigation was performed by means of DSC, using scanning rates between 2 and $20 \mathrm{~K} / \mathrm{min}$ in the temperature range from 260 to $330 \mathrm{~K}$. The sequential heating and cooling runs revealed the $I-N_{U}$ transition between 315 and $320 \mathrm{~K}$, depending on heating or cooling as well as on the rate used in each run. No peak could be detected in the range of temperatures where the $N_{U}-N_{B}$ transition was expected according to the previous IR and conoscopic measurements [16]. The DSC results can be seen in Figure 2 and Figure 3 for heating and cooling runs, respectively.

To perform a more thorough thermal study we employed ASC, using very slow scanning rates (2 to 3 orders of magnitude slower that the ones of DSC). We carried out a number of sequential heating and cooling runs, in the range between room temperature and $325 \mathrm{~K}$. Details about the ASC runs are shown in Table I. Concerning the scanning rates, it is noteworthy that they represent average values. In the vicinity of a phase transition the

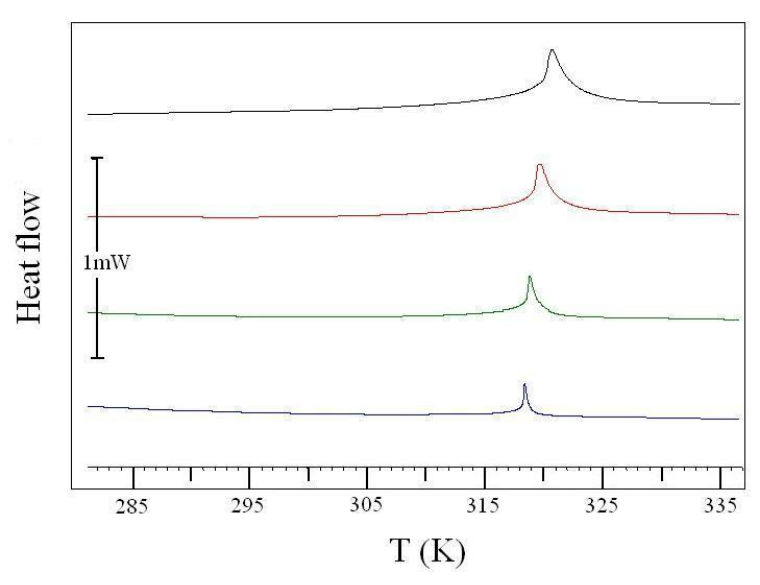

FIG. 2: DSC heating runs of $10,10,5$ and $2 \mathrm{~K} / \mathrm{min}$ (from top to bottom). Note: the thermographs have been shifted along the y-axis.

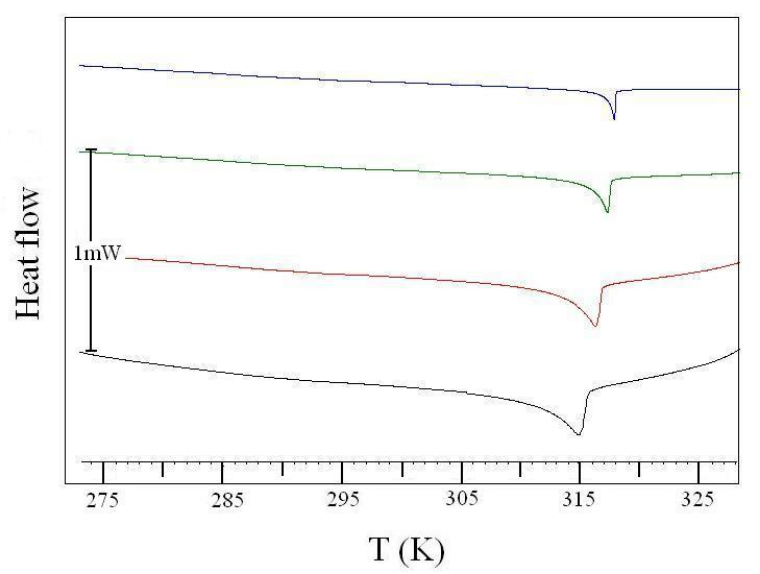

FIG. 3: DSC cooling runs of 2, 5, 10 and $10 \mathrm{~K} / \mathrm{min}$ (from top to bottom). Note: the thermographs have been shifted along the y-axis.

actual rate is slower since the (constant) applied power is spent on both temperature change and conversion between phases. Prior to cooling runs the samples were heated up to the $I$ phase and then stabilised to the initial temperature.

In Figure 4 the $C_{p}(T)$ curve is shown for the first heating run. The $I-N_{U}$ transition is clearly visible, but no heat capacity anomaly can be detected in temperatures around $310 \mathrm{~K}$, where the $N_{U}-N_{B}$ transition is expected according to the ref. [16]. The specific heat capacity and enthalpy temperature dependence in the area around the $I-N_{U}$ phase transition are shown on top and bottom of Figure 5, respectively. They are obtained from the second run (see Table I). The insets show the magnified data in a short range of $100 \mathrm{mK}$ around the transition. The $I-N_{U}$ phase transition was always found at the same 
TABLE I: The features of ASC scans performed for the DW16 compound.

\begin{tabular}{cccc}
\hline \hline Number of run & Type of run & T-range $(\mathrm{K})$ & Rate $(\mathrm{mK} / \mathrm{min})$ \\
\hline 1 & heating & $305.15-325.15$ & 4.5 \\
2 & cooling & $325.15-299.15$ & 2.5 \\
3 & cooling & $309.15-295.15$ & 2.5 \\
4 & cooling & $322.15-295.15$ & 2.5 \\
5 & heating & $311.15-323.15$ & 4.5 \\
6 & cooling & $313.15-303.15$ & 2.0 \\
7 & heating & $295.15-323.15$ & 56.8 \\
\hline
\end{tabular}

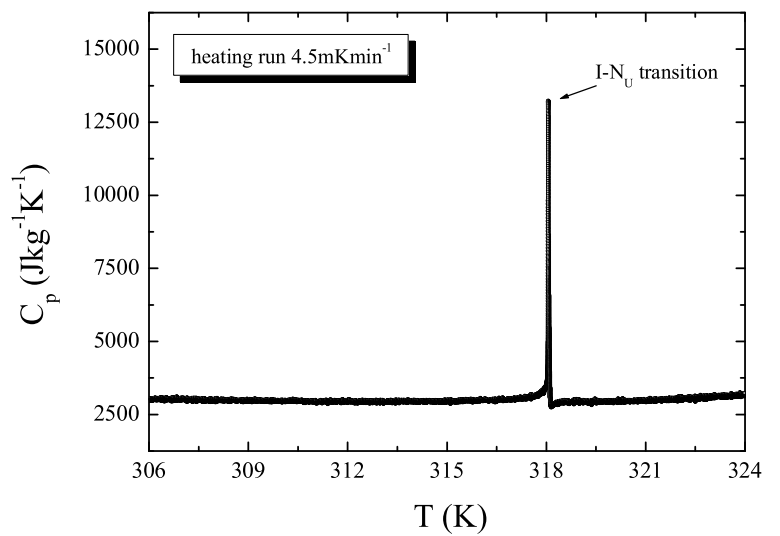

FIG. 4: Temperature profile of the specific heat capacity for the first heating run of DW-16. The $I-N_{U}$ transition occurs at $318.06 \mathrm{~K}$, but there is no visible trace of a $N_{U}-N_{B}$ transition.

temperature $T_{I N u}=318.06 \pm 0.01 \mathrm{~K}$ for the different heating and cooling runs, confirming the high stability of this compound. This transition is very weakly first order with a latent heat $L=0.20 \pm 0.02 \mathrm{~J} / \mathrm{g}$ released over a narrow temperature range of $22 \pm 2 \mathrm{mK}$. This latent heat is remarkably small, compared to the usually measured ones for other liquid crystals [35]. The total transition heat (latent heat plus pre-transitional increases) for the $I-N_{U}$ transition obtained by DSC and ASC are in very good agreement, yielding $\Delta H_{D S C}=1.3 \pm 0.1 \mathrm{~J} / \mathrm{g}$ and $\Delta H_{A S C}=1.27 \pm 0.05 \mathrm{~J} / \mathrm{g}$, respectively.

A schematic overview of the location of the observed peaks for all ASC runs is presented in Figure 6. In the temperature range where a $N_{U}-N_{B}$ transition was found in ref. [16], no specific heat capacity anomaly is observed in any of the runs here, as shown in Figure 7. The maximum $C_{p}$ noise in slow scans was never exceeding the \pm 1 $\%$ of the absolute values and, therefore, it can not be attributed to any kind of phase transition. The absence of a $N_{U}-N_{B}$ peak means that this transition must involve an extremely small enthalpy change, smaller than the experimental accuracy of $1 \mathrm{~mJ} / \mathrm{g}$ for our measurements.

Between the various runs of Figure 7 one may observe small differences in the slope of $C_{p}(T)$ curves. They occur
$\mathrm{T}(\mathrm{K})$

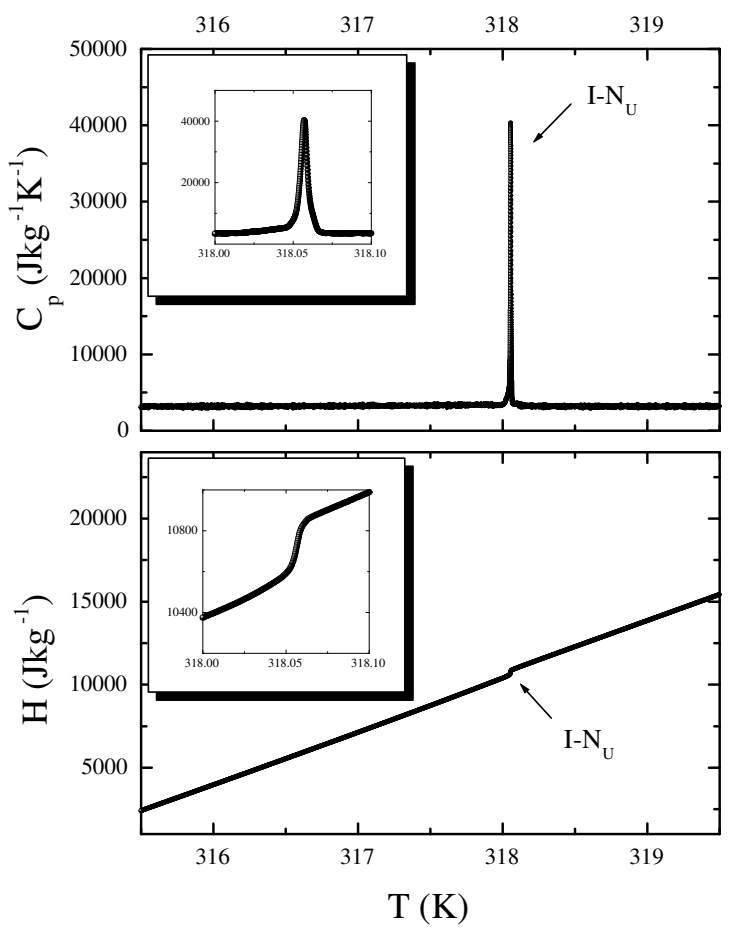

FIG. 5: Heat capacity (top) and enthalpy curves (bottom) for the $I-N_{U}$ phase transition, with blow ups of $100 \mathrm{mK}$ area around the transition temperature as insets. In the enthalpy curve a linear background is subtracted from the original data for clarity. The data plotted here correspond to the first cooling run (see Table I).

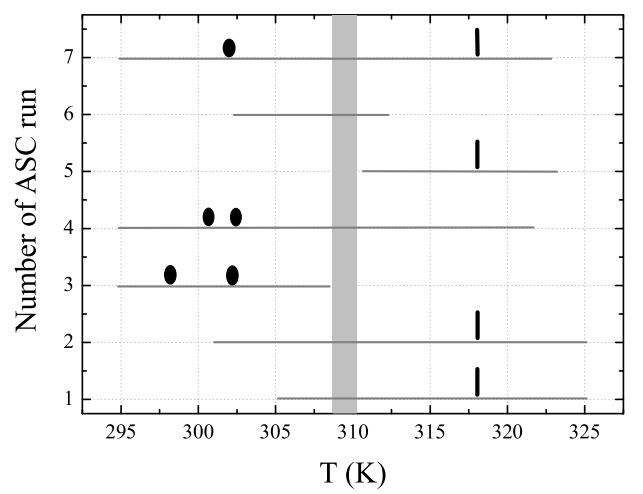

FIG. 6: A schematic overview of the ASC runs and the observed peaks. The spikes correspond to the $I-N_{U}$ transition temperature and the ellipsoidal symbols to the solidification/melting of DW-16. The shady area, in light-grey colour, represents the temperature range where a signature of $N_{U}-N_{B}$ transition was expected according to Ref. [16]. 


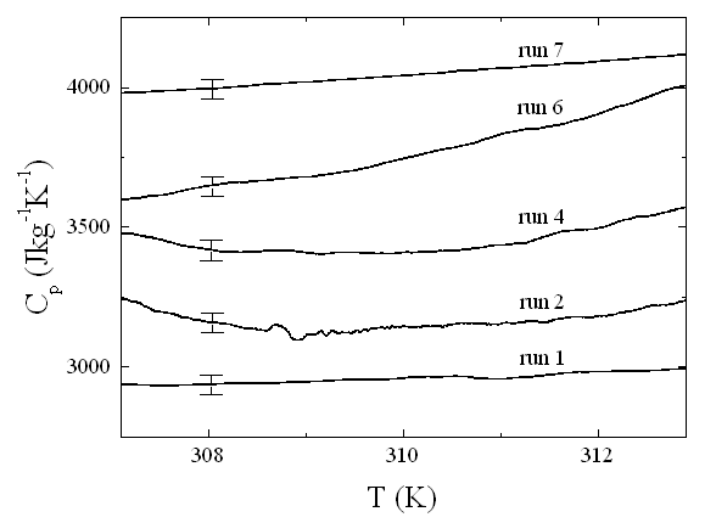

FIG. 7: A detailed plot of specific heat capacity in the temperature range between 307 and $313 \mathrm{~K}$, where a trace of $N_{U}-N_{B}$ transition is expected. The different data sets have been shifted along the y-axis for clarity. For each case, the number of run corresponds to the number written in Table I. The changes of $C_{p}$ values are negligible, as it can be seen in comparison to the artificially added error bars of $\pm 1 \%$.

because of approaching towards the vicinal phase transitions, namely the $I-N_{U}$ transition in higher temperatures and the solidification of the sample in lower temperatures (for this transition see the text later on). The data were collected upon heating or cooling and with different scanning rates, as it can be identified from the Table I. As a consequence, the transition temperatures and/or the pre-transitional effects can be sufficiently affected, resulting to slight changes of $C_{p}(T)$ background further away from transition temperatures. Dissimilarities in the shape (eg. sharpness and pre-transitional wings) of $C_{p}$ anomalies are observable even among different very slow runs, as it comes out from a comparison of the stable $I-N_{U}$ peak between heating of Figure 4 and cooling of Figure 5 .

Below $305 \mathrm{~K}$ and close to room temperature strongly first order transitions have been observed on both heating and cooling runs. These transitions were very dependent on the scanning rate and the thermal history of the sample, and they are attributed to the solidification (upon cooling) and melting (upon heating) of the sample. As it can be seen in Figure 8, in case of slow cooling runs two separate but still relatively wide strongly first order anomalies are observed. Upon the last fast (in terms of ASC) heating run (see Table I) these two peaks are again observed, but unified as a significantly broadened $C_{p}$ anomaly, that cannot be easily distinguished if plotted in the same scale with the slow cooling runs of Figure 8. The reason why these transitions are not observed in some of the DSC runs at similar temperatures is possibly caused by supercooling, due to the very fast rates, and subsequent heating of the sample.

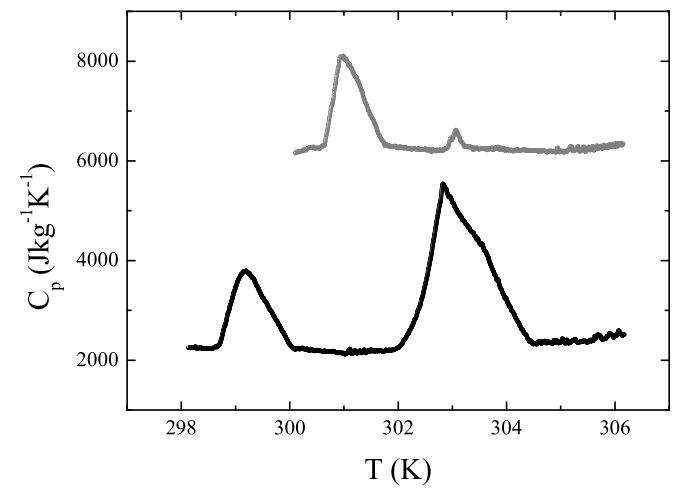

FIG. 8: The specific heat capacity profiles in low temperatures, for the second (black symbols) and third (grey symbols) cooling run (third and fourth run in Table I, respectively), showing the first order solidification/melting transitions of DW-16. The latter curve (third cooling run) has been shifted along the y-axis for lucid representation.

\section{SUMMARY}

We have studied experimentally the phase transitions of an organo-siloxane tetrapode, that was reported to exhibit a $N_{B}$ phase [16]. In sequential heating and cooling runs, and within a range of very fast (DSC measurements) down to very slow (ASC measurements) scanning rates, the $N_{U}-N_{B}$ phase transition was not discernible. From our measurements it is concluded that a very small transition heat must be related to the $N_{U}-N_{B}$ transition, which for DW-16 should be less than $1 \mathrm{~mJ} / \mathrm{g}$. This observation is in accordance with previous measurements for a bent-core mesogen, where the transition flaunting under x-rays did not leave any thermal trace in DSC thermographs [14]. On the other hand, the $I-N_{U}$ transition was very stable and weakly first order with a rather small latent heat of $0.20 \pm 0.02 \mathrm{~J} / \mathrm{g}$ compared to other compounds [35].

\section{ACKNOWLEDGEMENTS}

This research was financially supported by the Fund for Scientific Research Flanders (FWO, Project G. 0230.07). G.C. acknowledges the receipt of postdoctoral fellowship of FWO and of Research Fund of K. U. Leuven. G.H.M. and D.A. thank Mrs. J. Haley for the DSC measurements and acknowledge the support through the E.U. Project "BIND". 
[3] J. P. Straley, Phys. Rev. A 10, 1881 (1974).

[4] L. J. Yu and A. Saupe, Phys. Rev. Lett. 45, 1000 (1980).

[5] G. R. Luckhurst, Thin Solid Films. 393, 40 (2001).

[6] S. Chandrasekhar, G. G. Nair, K. Praefcke, and D. Singer, Mol. Cryst. Liq. Cryst. 288, 7 (1996).

[7] S. Chandrasekhar, G. G. Nair, D. S. Shankar Rao, and S. Krishna Prasad, Liq. Cryst 24, 67 (1998).

[8] K. Severing and K. Saalwachter, Phys. Rev. Lett. 92, 125501 (2004).

[9] K. Severing, E. Stibal-Fischer, A. Hasenhindl, H. Finkelmann, and K. Saalwachter, J. Phys. Chem. B 110, 15680 (2006).

[10] B. R. Acharya, A. Primak, T. J. Digenmans, E. T. Samulski, and S. Kumar, Pramana 61, 231 (2003).

[11] L. A. Madsen, T. J. Dingenmans, M. Nakata, and E. T. Samulski, Phys. Rev. Lett. 92, 145505 (2004).

[12] B. R. Acharya, A. Primak, and S. Kumar, Phys. Rev. Lett. 92, 145506 (2004).

[13] C. V. Yelamaggad, S. Krishna Prasad, G. G. Nair, I. S. Shashikala, D. S. Shankar Rao, G. V. Lobo, and S. Chandrasekhar, Angew. Chem. Int. Ed. 43, 3429 (2004).

[14] V. Prasad, S. W. Kang, K. A. Suresh, L. Joshi, Q. Wang, and S. Kumar, J. Am. Chem. Soc. 127, 17224 (2005).

[15] S. Krishna Prasad, G. G. Nair, D. S. Shankar Rao, G. V. Lobo, I. Shashikala, and C. V. Yelemaggad, Mol. Cryst. Liq. Cryst. 437, 211 (2005).

[16] K. Merkel, A. Kocot, J. K. Vij, R. Korlacki, G. H. Mehl, and T. Meyer, Phys. Rev. Lett. 93, 237801 (2004).

[17] J. L. Figuerinhas, C. Cruz, D. Filip, G. Feio, A. C. Ribeiro, Y. Frere, T. Meyer, and G. H. Mehl, Phys. Rev. Lett. 94, 107802 (2005).

[18] K. Neupane, S. W. Wang, S. Sharma, D. Carney, T. Meyer, G. H. Mehl, D. Allender, S. Kumar, and S. Sprunt, Phys. Rev. Lett. 97, 207802 (2006).
[19] G. R. Luckhurst, Nature 430, 413 (2004).

[20] J. H. Lee, T. K. Lim, W. T. Kim, and J. I. Jin, J. Appl. Phys. 101, 034105 (2007).

[21] R. Berardi, L. Muccioli, and C. Zannoni, J. Chem. Phys. 128, 024905 (2008).

[22] A. M. Sonnet, E. G. Virga, and G. E. Durand, Phys. Rev. E 67, 061701 (2003).

[23] B. Mettout, Phys. Rev. E 72, 031706 (2005).

[24] M. A. Bates and G. R. Luckhurst, Phys. Rev. E 72, $051702(2005)$

[25] L. longa, P. Grzybowski, S. Romano, and E. G. Virga, Phys. Rev. E 71, 051714 (2005).

[26] G. De Matteis and E. G. Virga, Phys. Rev. E 71, 061703 (2005).

[27] G. De Matteis, S. Romano, and E. G. Virga, Phys. Rev. E 72, 041706 (2005).

[28] F. Bisi, E. G. Virga, E. C. Gartland, Jr., G. De Matteis, A. M. Sonnet, and G. E. Durand, Phys. Rev. E 73, 051709 (2006)

[29] J. Peláez and M. R. Wilson, Phys. Rev. Lett. 97, 267801 (2006).

[30] F. Bisi, S. Romano, and E. G. Virga, Phys. Rev. E 75, 041705 (2007).

[31] L. longa, G. Pajak, and T. Wydro, Phys. Rev. E 76, 011703 (2007).

[32] A. G. Vanakaras and D. J. Photinos, J. Chem. Phys 128, $124512(2008)$

[33] J. Thoen, H. Marynissen, and W. Van Dael, Phys. Rev. A 26, 2886 (1982)

[34] J. Thoen, Int. J. Mod. Phys. B 9, 2157 (1995).

[35] B. V. Roie, J. Leys, K. Denolf, C. Glorieux, G. Pitsi, and J. Thoen, Phys. Rev. E 72, 041702 (2005). 